MATEC Web of Conferences 40, 02023 (2016)

DOI: $10.1051 /$ matecconf $/ 20164002023$

(c) Owned by the authors, published by EDP Sciences, 2016

\title{
Characterization and Development of a KL Driving Cycle for PHERB Powertrain
}

\author{
A. R. Salisa ${ }^{1}$, W. H. Atiq ${ }^{1}$, and J. S. Norbakyah ${ }^{1}$ \\ ${ }^{1}$ School of Ocean Engineering, Universiti Malaysia Terengganu, 21030 Kuala \\ Terengganu, Terengganu, Malaysia
}

\begin{abstract}
This paper presents the result that being obtained by development of Kenyir Lake driving cycle for a plugin hybrid electric recreational boat (PHERB) powertrain. This research is conducted by using on board measurement techniques; second-by-second driving speed data correlate to time is collected by using global positioning system of the boat along selected route. A combination of batteries and ultracapacitor pack are used for hybrid energy storage system in PHERB powertrain which is good for driving performance and energy efficiency. The developed driving cycle contains a $2294 \mathrm{~s}$ speed time series, with a distance of $8.19 \mathrm{~km}$, and an average and a maximum speed of 12.85 $\mathrm{km} / \mathrm{h}$ and $36.91 \mathrm{~km} / \mathrm{h}$, respectively. The characteristics of KL driving cycle are compared with existing standard drive cycles. The results obtained from this analysis are within reasonable range and satisfactory.
\end{abstract}

\section{Introduction}

In recent years, global warming has become a major concern among all researchers, scientist and academicians. Global warming is associated to steady increase of temperature at Earth's atmosphere. Motor vehicle is one of the main contributors of global warming. Rapid increment in number of vehicles has result in high fuel consumption, which later releases emission. A huge amount of emission released had cause increased volumes of carbon dioxide and greenhouse gas. Therefore, there is a need to conduct a research on method to combat global warming by reducing the fuel consumption and emission. Driving Cycle is a core element in vehicle emission model and powertrain input [1]. A driving cycle is a representation of a speed-time data profile of vehicle for a particular area or city [2]. It is broadly used to determining fuel consumption pattern and estimate vehicle air pollutant emissions for a vehicle in specific place. Driving cycle contain four sequence of vehicle operating condition (idle, acceleration, cruise and deceleration) and is regarded as a signature of driving pattern of a particular place. The aim of this study is to acquire a better understanding of driving characteristics (average speed, running speed, acceleration, deceleration, mean length, time proportion of idling, cruise, acceleration, deceleration, root mean square of acceleration and deceleration, and acceleration energy per kilometer) in KL because the driving cycle at KL is still not exists. The driving cycle that has been developed will be implemented in Plug-In-Hybrid Electric Recreational Boat (PHERB) powertrain. Then, the characteristics of a developed KL river driving cycle for Plug-in Hybrid Electric Recreational Boat (PHERB) powertrain is compared with the existing driving cycles.

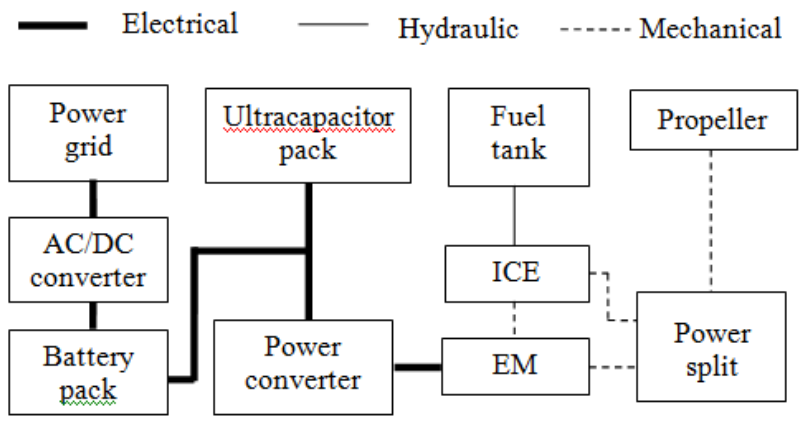

Figure. 1. A schematic illustration of the proposed seriesparallel PHERB powertrain.

\section{Driving Cycle Development}

To construct a driving cycle, three stages were executed in this research which are route selection, data collection and drive cycle construction. In terms of route selection, the selected route is the most common route used by tourist guide to travel along small landmass at KL. For the purpose of data collection, there are three techniques that are available for researcher to collect the speed-time data [3]. First, chase boat technique, where a boat equipped with instrument and sensor is used to record the speedtime data of target boat. The second method is on-board measurement techniques which rely solely on GPS (Global Positioning System) to collect each single second 
of speed-time data. The third method is combination of both techniques. However, this study only adopts the second method since there are insufficient expert boat driver to undertake the chase boat method. Besides, the second method can minimize the possibility of boat accident. According to the past study [4 -5], 12 variables are used to analyse and characterize KL driving cycle speed-time data as listed in Table I. Fig. 2 present the flowchart regarding the stages of data analysis and development of driving cycle. The selected route for KL driving cycle development is illustrated in Fig. 3.

Table 1. Variables Used in KL Driving Cycle Analysis

\begin{tabular}{|c|c|c|c|}
\hline No & Variable & Unit & Formula \\
\hline 1 & Average Speed, $v_{1}$ & $\mathrm{~km} / \mathrm{h}$ & $v_{-} a v g=3.6 \frac{d i s t}{T_{\text {total }}}$ \\
\hline 2 & Average Running Speed, $v_{2}$ & $\mathrm{~km} / \mathrm{h}$ & $v_{-}$run $=3.6 \frac{\text { dist }}{T_{\text {drive }}}$ \\
\hline 3 & Average Acceleration, $a$ & $\mathrm{~m} / \mathrm{s}^{2}$ & $a_{-} a v g=\left(\sum_{i=1}^{n}\left\{\begin{array}{ll}1\left(a_{i}>0\right) \\
0(\mathrm{else})\end{array}\right)^{-1} \sum_{i=1}^{n} \begin{array}{ll}1 & \left(\mathrm{a}_{\mathrm{i}}>0\right)\end{array}\right.$ \\
\hline 4 & Average Deceleration, $d$ & $\mathrm{~m} / \mathrm{s}^{2}$ & $d_{-} a v g=\left(\sum_{i=1}^{n}\left\{\begin{array}{ll}1\left(a_{i}<0\right) \\
0(\mathrm{else})\end{array}\right)^{-1} \sum_{i=1}^{n} \begin{array}{ll}1 & \left(\mathrm{a}_{\mathrm{i}}<0\right) \\
0 & (\mathrm{else})\end{array}\right.$ \\
\hline 5 & Mean Length of a driving period, $c$ & $\mathrm{~s}$ & $T_{\text {total }}=t_{2}-t_{1}+\sum_{i=2}^{n}\left(t_{i}-t_{i-1}\right)$ \\
\hline 6 & Time Proportion of Idling, $P_{i}$ & $\%$ & $\%$ drive $=\frac{T_{\text {drive }}}{T_{\text {total }}}$ \\
\hline 7 & Time Proportion of Cruise, $P_{c}$ & $\%$ & $\%$ cruise $=\frac{T_{\text {cruise }}}{T_{\text {total }}}$ \\
\hline 8 & Time Proportion of Acceleration, $p_{a}$ & $\%$ & $\%$ oacc $=\frac{T_{a c c}}{T_{\text {total }}}$ \\
\hline 9 & Time Proportion of Deceleration, $P_{d}$ & $\%$ & $\%$ dec $=\frac{T_{\text {dec }}}{T_{\text {total }}}$ \\
\hline 10 & $\begin{array}{l}\text { Average number of acceleration-deceleration } \\
\text { changes within one driving period, } M\end{array}$ & & - \\
\hline 11 & Root Mean Square of Acceleration, $R M S$ & $\mathrm{~m} / \mathrm{s}^{2}$ & $R M S=\sqrt{\frac{1}{T_{\text {total }}}} \sum_{i=1}^{n} a_{i}^{2}$ \\
\hline 12 & Acceleration Energy per Kilometer, $K P E$ & $\mathrm{~m} / \mathrm{s}^{2}$ & $P K E=\frac{1}{\text { dist }} \sum_{i=2}^{n} \begin{cases}v_{i}^{2}-v_{i-1}^{2} & \left(v_{i}>v_{i-1}\right) \\
& (\text { else })\end{cases}$ \\
\hline
\end{tabular}




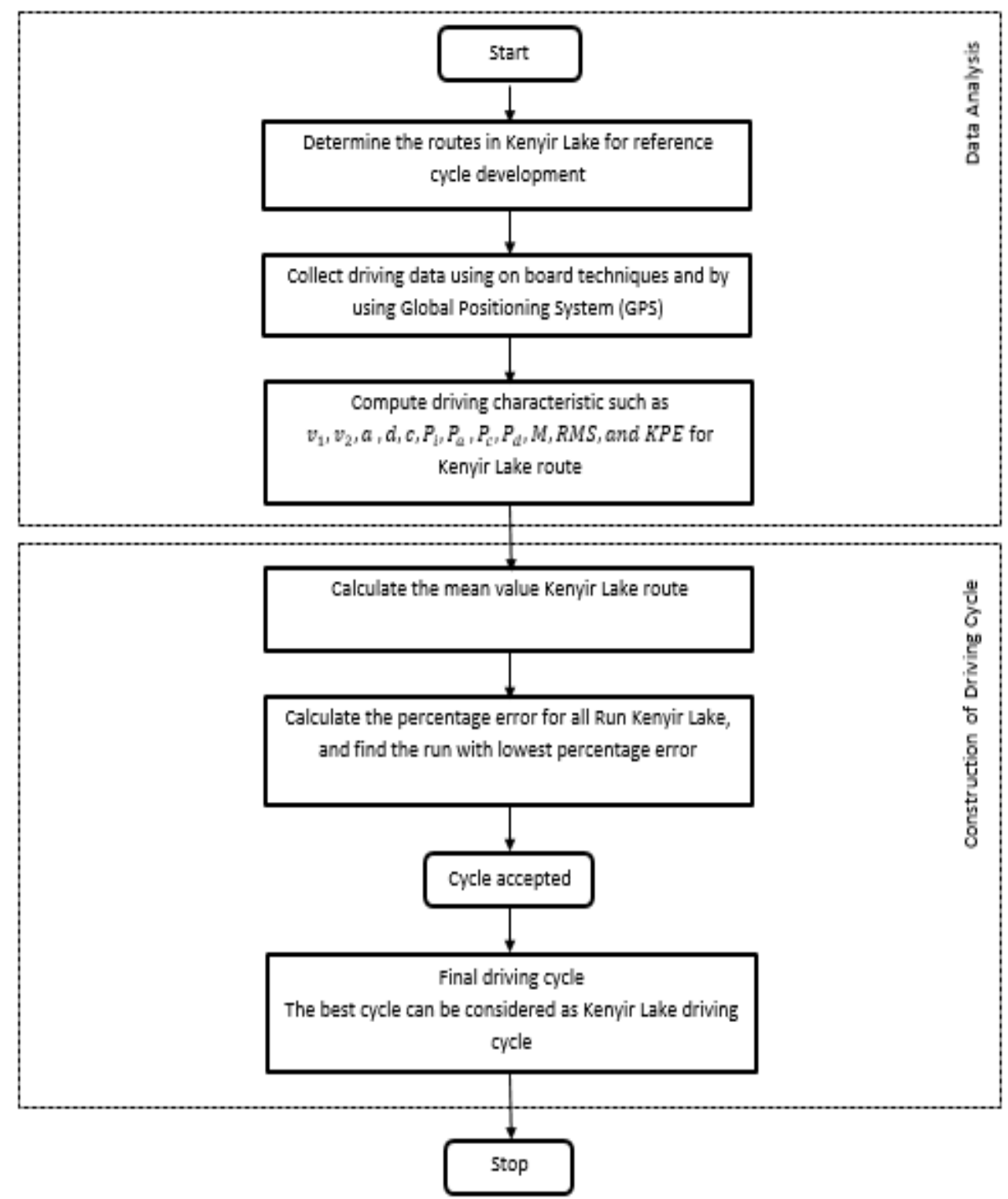

Figure. 2. Flow chart of Kenyir Lake Driving Cycle Development

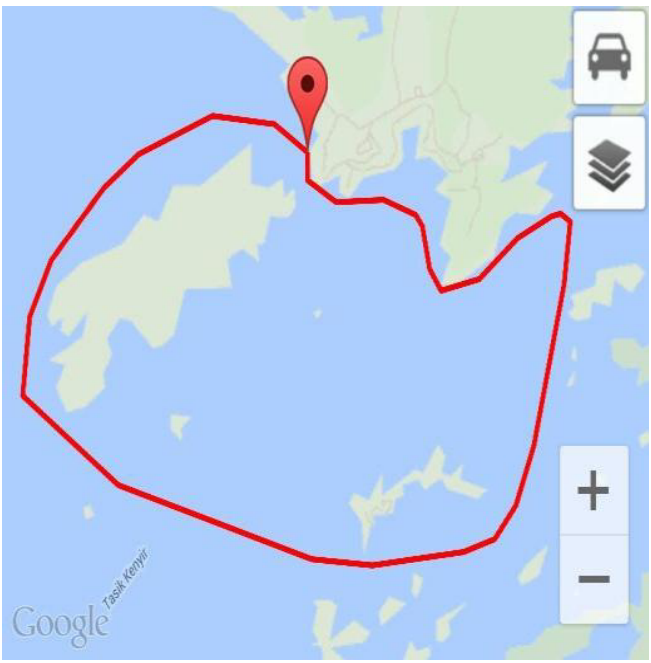

Figure. 3. The selected route of Kenyir Lake 


\section{Data Analysis}

The data is collected during the period of 10:00-12:00p.m for 20 days on April 2015. One run is handled to record one data per day. In the end 20 run was collected. SpeedTime data is collected by using Global Positioning System along the selected route around KL. The value of 12 data variables which has been used in conducting the speed-time data analysis is shown in Table 2. The mean values in Table 3 are obtained from Table 2. The mean value from table 3 has been calculated by summation of data in table 2 which was divided by 20 runs. After the mean value is gained, the percentage error for all run is calculated in Table 4 . In the Table 5 it shown the most minimum percentage error is from Run 9 that has been calculated in the Table 4. Run 9 is the one with the smallest percentage error is considered as the best KL drive cycle. The average speed for KL is $12.85 \mathrm{~km} / \mathrm{h}$ and the average running speed is $12.91 \mathrm{~km} / \mathrm{h}$. The results indicated that the speed is very slow. For complete circulation at $\mathrm{KL}$, the rate for acceleration and deceleration is equal which is 0.35.The four vehicle operating modes is calculated. The time proportion of idling, time proportion of cruising, time proportion of acceleration and time proportion of deceleration are $0.44 \%, 0.70 \%, 49.54 \%$ and $49.37 \%$ respectively. The value of total time and mean length of driving period is equal, that is $2294 \mathrm{~s}$. When the driving period is close or equal to total time, it showed that the driving period is high, and it indicates that the route is clear and there is no obstacle along the path. The Root Mean Square for this run is 0.43 and the Acceleration Energy per Kilometer is 0.35 . The best KL driving cycle is constructed as presented in Fig. 4.

Table 2. KL driving cycle data analysis

\begin{tabular}{|c|c|c|c|c|c|c|c|c|c|c|c|c|}
\hline Criterion & $\begin{array}{c}v_{1} \\
(\mathbf{k m} / \mathbf{h})\end{array}$ & $\begin{array}{c}v_{2} \\
(\mathbf{k m} / \mathbf{h})\end{array}$ & $\begin{array}{c}a \\
\left(\mathbf{m} / \mathbf{s}^{2}\right)\end{array}$ & $\begin{array}{c}d \\
\left(\mathbf{m} / \mathbf{s}^{2}\right)\end{array}$ & $\begin{array}{l}c \\
\text { (s) }\end{array}$ & $\begin{array}{l}P_{i} \\
(\%)\end{array}$ & $\begin{array}{l}P_{c} \\
\text { (\%) }\end{array}$ & $\begin{array}{l}p_{a} \\
(\%)\end{array}$ & $\begin{array}{l}P_{d} \\
(\%)\end{array}$ & $\mathbf{M}$ & $\begin{array}{l}R M S \\
\left(\mathbf{m} / \mathbf{s}^{2}\right)\end{array}$ & $\begin{array}{l}K P E \\
\left(\mathbf{m} / \mathbf{s}^{2}\right)\end{array}$ \\
\hline $\mathrm{j}$ & 1 & 2 & 3 & 4 & 5 & 6 & 7 & 8 & 9 & 10 & 11 & 12 \\
\hline Run 1 & 12.93 & 13.00 & 0.26 & 0.27 & 2275 & 0.53 & 0.04 & 50.22 & 49.34 & 1.00 & 0.34 & 0.28 \\
\hline Run 2 & 12.99 & 13.07 & 0.28 & 0.29 & 2307 & 0.61 & 0.04 & 50.61 & 48.87 & 0.99 & 0.36 & 0.28 \\
\hline Run 3 & 12.85 & 12.90 & 0.27 & 0.27 & 2295 & 0.39 & 0.04 & 49.74 & 49.96 & 1.00 & 0.35 & 0.28 \\
\hline Run 4 & 12.89 & 12.98 & 0.26 & 0.26 & 2252 & 0.67 & 0.44 & 49.67 & 49.27 & 0.99 & 0.34 & 0.27 \\
\hline Run ...... & ..... & ..... & ..... & ..... & ..... & $\ldots .$. & $\ldots .$. & ..... & ...... & $\ldots \ldots$ & ..... & ...... \\
\hline Run 9 & 12.85 & 12.91 & 0.35 & 0.35 & 2294 & 0.44 & 0.70 & 49.54 & 49.37 & 0.99 & 0.43 & 0.35 \\
\hline Run ...... & ..... & ..... & ..... & ..... & ..... & $\ldots .$. & $\ldots .$. & ..... & ..... & $\ldots .$. & ..... & $\ldots .$. \\
\hline Run 17 & 12.93 & 12.98 & 0.36 & 0.35 & 2274 & 0.44 & 0.22 & 49.10 & 50.29 & 0.99 & 0.46 & 0.37 \\
\hline Run 18 & 13.02 & 13.08 & 0.36 & 0.37 & 2299 & 0.48 & 0.74 & 50.39 & 48.43 & 0.99 & 0.45 & 0.37 \\
\hline Run 19 & 12.85 & 12.92 & 0.35 & 0.34 & 2264 & 0.57 & 1.72 & 48.17 & 49.58 & 0.98 & 0.44 & 0.34 \\
\hline Run 20 & 12.89 & 12.95 & 0.36 & 0.36 & 2280 & 0.48 & 0.31 & 50.24 & 49.01 & 0.99 & 0.45 & 0.37 \\
\hline
\end{tabular}

Table 3. Mean values of the assessment parameters of grouped runs

\begin{tabular}{|c|c|c|c|c|c|c|c|c|c|c|c|c|}
\hline Criterion & $\begin{array}{c}v_{1} \\
(\mathbf{k m} / \mathbf{h})\end{array}$ & $\begin{array}{c}v_{2} \\
(\mathbf{k m} / \mathbf{h})\end{array}$ & $\begin{array}{c}a \\
\left(\mathbf{m} / \mathbf{s}^{\mathbf{2}}\right)\end{array}$ & $\begin{array}{c}d \\
\left(\mathbf{m} / \mathbf{s}^{\mathbf{2}}\right)\end{array}$ & $\begin{array}{c}c \\
\mathbf{( s )}\end{array}$ & $\begin{array}{c}P_{i} \\
\mathbf{( \% )}\end{array}$ & $\begin{array}{c}P_{c} \\
\mathbf{( \% )}\end{array}$ & $\begin{array}{c}p_{a} \\
\mathbf{( \% )}\end{array}$ & $\begin{array}{c}P_{d} \\
(\mathbf{\%})\end{array}$ & $\mathbf{M}$ & $\begin{array}{c}R M S \\
\left(\mathbf{m} / \mathbf{s}^{2}\right)\end{array}$ & $\begin{array}{c}K P E \\
\left(\mathbf{m} / \mathbf{s}^{\mathbf{2}}\right)\end{array}$ \\
\hline $\mathrm{j}$ & 1 & 2 & 3 & 4 & 5 & 6 & 7 & 8 & 9 & 10 & 11 & 12 \\
\hline $\begin{array}{c}\text { Mean } \\
\text { Value } \\
\text { Route 1 } \\
\text { (run 1 } \\
\text { run 20) }\end{array}$ & 12.92 & 12.99 & 0.33 & 0.33 & 2280.45 & 0.50 & 0.56 & 47.25 & 49.33 & 0.99 & 0.42 & 0.34 \\
\hline
\end{tabular}


Table 4. Percentage difference relative to target summary statistics

\begin{tabular}{|c|c|c|c|c|c|c|c|c|c|c|c|c|c|}
\hline $\begin{array}{c}\text { Criterio } \\
\mathbf{n}\end{array}$ & $\begin{array}{c}v_{1} \\
(\mathrm{~km} / \mathrm{h} \\
)\end{array}$ & $\begin{array}{c}v_{2} \\
(\mathrm{~km} / \mathrm{h} \\
)\end{array}$ & $\begin{array}{c}a \\
(\mathrm{~m} / \mathrm{s} 2 \\
)\end{array}$ & $\begin{array}{c}d \\
(\mathrm{~m} / \mathrm{s} 2 \\
)\end{array}$ & $\begin{array}{l}c \\
(\mathbf{s})\end{array}$ & $\begin{array}{c}P_{i} \\
(\mathbf{\%}) \\
\end{array}$ & $\begin{array}{l}P_{c} \\
(\mathbf{\%}) \\
\end{array}$ & $\begin{array}{c}p_{a} \\
\mathbf{( \% )}\end{array}$ & $\begin{array}{l}P_{d} \\
(\%)\end{array}$ & M & $\begin{array}{l}R M S \\
(\mathbf{m} / \mathbf{s} \mathbf{2}) \\
\end{array}$ & $\begin{array}{l}K P E \\
(\mathbf{m} / \mathbf{s} \mathbf{2}) \\
\end{array}$ & $\begin{array}{c}\text { Total } \\
\text { Error } \\
(\%) \\
\end{array}$ \\
\hline $\mathrm{J}$ & 1 & 2 & 3 & 4 & 5 & 6 & 7 & 8 & 9 & 10 & 11 & 12 & \\
\hline $\begin{array}{l}\text { Mean } \\
\text { Value }\end{array}$ & 12.92 & 12.99 & 0.33 & 0.33 & $\begin{array}{l}2280.4 \\
5 \\
\end{array}$ & 0.50 & 0.56 & $\begin{array}{c}47.2 \\
5\end{array}$ & $\begin{array}{c}49.3 \\
3\end{array}$ & $\begin{array}{l}0.9 \\
9\end{array}$ & 0.42 & 0.34 & \\
\hline Run 1 & 0.08 & 0.08 & 21.21 & 18.18 & 0.24 & 6.00 & 92.86 & 6.29 & 0.02 & $\begin{array}{c}1.0 \\
1\end{array}$ & 19.05 & 17.65 & $\begin{array}{c}182.6 \\
7\end{array}$ \\
\hline Run 2 & 0.54 & 0.62 & 15.15 & 12.12 & 1.16 & $\begin{array}{c}22.0 \\
0 \\
\end{array}$ & 92.86 & 7.11 & 0.93 & 0 & 14.29 & 17.65 & $\begin{array}{c}184.4 \\
3\end{array}$ \\
\hline Run 3 & 0.54 & 0.69 & 18.18 & 18.18 & 0.64 & $\begin{array}{c}22.0 \\
0 \\
\end{array}$ & 92.86 & 5.27 & 1.28 & $\begin{array}{c}1.0 \\
1\end{array}$ & 16.67 & 17.65 & $\begin{array}{c}194.9 \\
7\end{array}$ \\
\hline Run 4 & 0.23 & 0.08 & 21.21 & 21.21 & 1.25 & $\begin{array}{c}34.0 \\
0 \\
\end{array}$ & 21.43 & 5.12 & 0.12 & 0 & 19.05 & 20.59 & $\begin{array}{c}144.2 \\
9\end{array}$ \\
\hline Run ...... & $\ldots \ldots$ & $\ldots \ldots$ & $\ldots \ldots$ & $\ldots \ldots$ & $\ldots \ldots$ & $\ldots \ldots$ & $\ldots \ldots$ & $\ldots .$. & $\ldots \ldots$ & $\ldots \ldots$ & $\ldots \ldots$ & $\ldots \ldots$ & $\ldots \ldots$ \\
\hline Run 9 & 0.54 & 0.62 & 6.06 & 6.06 & 0.59 & $\begin{array}{c}12.0 \\
0 \\
\end{array}$ & 25.00 & 4.85 & 0.08 & 0 & 2.38 & 2.94 & 61.12 \\
\hline Run ...... & $\cdots \cdots$ & $\ldots \ldots$ & $\cdots \cdots$ & $\cdots \cdots$ & $\cdots \cdots$ & $\cdots \cdots$ & $\cdots \cdots$ & $\cdots \cdots$ & $\cdots \cdots$ & $\cdots \cdots$ & $\ldots \ldots$ & $\ldots \ldots$ & $\cdots \cdots$ \\
\hline Run 16 & 0 & 0.08 & 9.09 & 12.12 & 0.37 & $\begin{array}{c}12.0 \\
0 \\
\end{array}$ & 55.36 & 5.08 & 0.51 & 0 & 9.52 & 5.88 & $\begin{array}{c}110.0 \\
1\end{array}$ \\
\hline Run 17 & 0.08 & 0.08 & 9.09 & 6.06 & 0.28 & $\begin{array}{c}12.0 \\
0 \\
\end{array}$ & 60.71 & 3.92 & 1.95 & 0 & 9.52 & 8.82 & $\begin{array}{c}112.5 \\
1\end{array}$ \\
\hline Run 18 & 0.77 & 0.69 & 9.09 & 12.12 & 0.81 & 4.00 & 32.14 & 6.65 & 1.82 & 0 & 7.14 & 8.82 & 84.05 \\
\hline Run 19 & 0.54 & 0.54 & 6.06 & 3.03 & 0.72 & $\begin{array}{c}14.0 \\
0 \\
\end{array}$ & $\begin{array}{c}207.1 \\
4 \\
\end{array}$ & 1.95 & 0.51 & $\begin{array}{c}1.0 \\
1 \\
\end{array}$ & 4.76 & 0 & $\begin{array}{c}240.2 \\
6\end{array}$ \\
\hline Run 20 & 0.23 & 0.31 & 9.09 & 9.09 & 0.02 & 4.00 & 44.64 & 6.33 & 0.65 & 0 & 7.14 & 8.82 & 90.32 \\
\hline
\end{tabular}

Table 5. Minimum Percentage Error of the Assessment Parameters of grouped runs

\begin{tabular}{|c|c|c|c|c|c|c|c|c|c|c|c|c|c|}
\hline Criterion & $\begin{array}{c}v_{1} \\
(\mathbf{k m} / \mathbf{h})\end{array}$ & $\begin{array}{c}v_{2} \\
(\mathbf{k m} / \mathbf{h})\end{array}$ & $\begin{array}{c}a \\
\left(\mathbf{m} / \mathbf{s}^{\mathbf{2}}\right)\end{array}$ & $\begin{array}{c}d \\
\left(\mathbf{m} / \mathbf{s}^{\mathbf{2}}\right)\end{array}$ & $\begin{array}{c}c \\
(\mathbf{s})\end{array}$ & $\begin{array}{c}P_{i} \\
\mathbf{( \% )}\end{array}$ & $\begin{array}{c}P_{c} \\
(\mathbf{\%})\end{array}$ & $\begin{array}{c}p_{a} \\
\mathbf{( \% )}\end{array}$ & $\begin{array}{c}P_{d} \\
(\mathbf{\%})\end{array}$ & $\mathbf{M}$ & $\begin{array}{c}R M S \\
\left(\mathbf{m} / \mathbf{s}^{\mathbf{2}}\right)\end{array}$ & $\begin{array}{c}K P E \\
\left(\mathbf{m} / \mathbf{s}^{\mathbf{2}}\right)\end{array}$ & $\begin{array}{c}\text { Total Error } \\
\mathbf{( \% )}\end{array}$ \\
\hline $\mathrm{J}$ & 1 & 2 & 3 & 4 & 5 & 6 & 7 & 8 & 9 & 10 & 11 & 12 & \\
\hline Run 9 & 0.54 & 0.62 & 6.06 & 6.06 & 0.59 & 12.00 & 25.00 & 4.85 & 0.08 & 0 & 2.38 & 2.94 & 61.12 \\
\hline
\end{tabular}




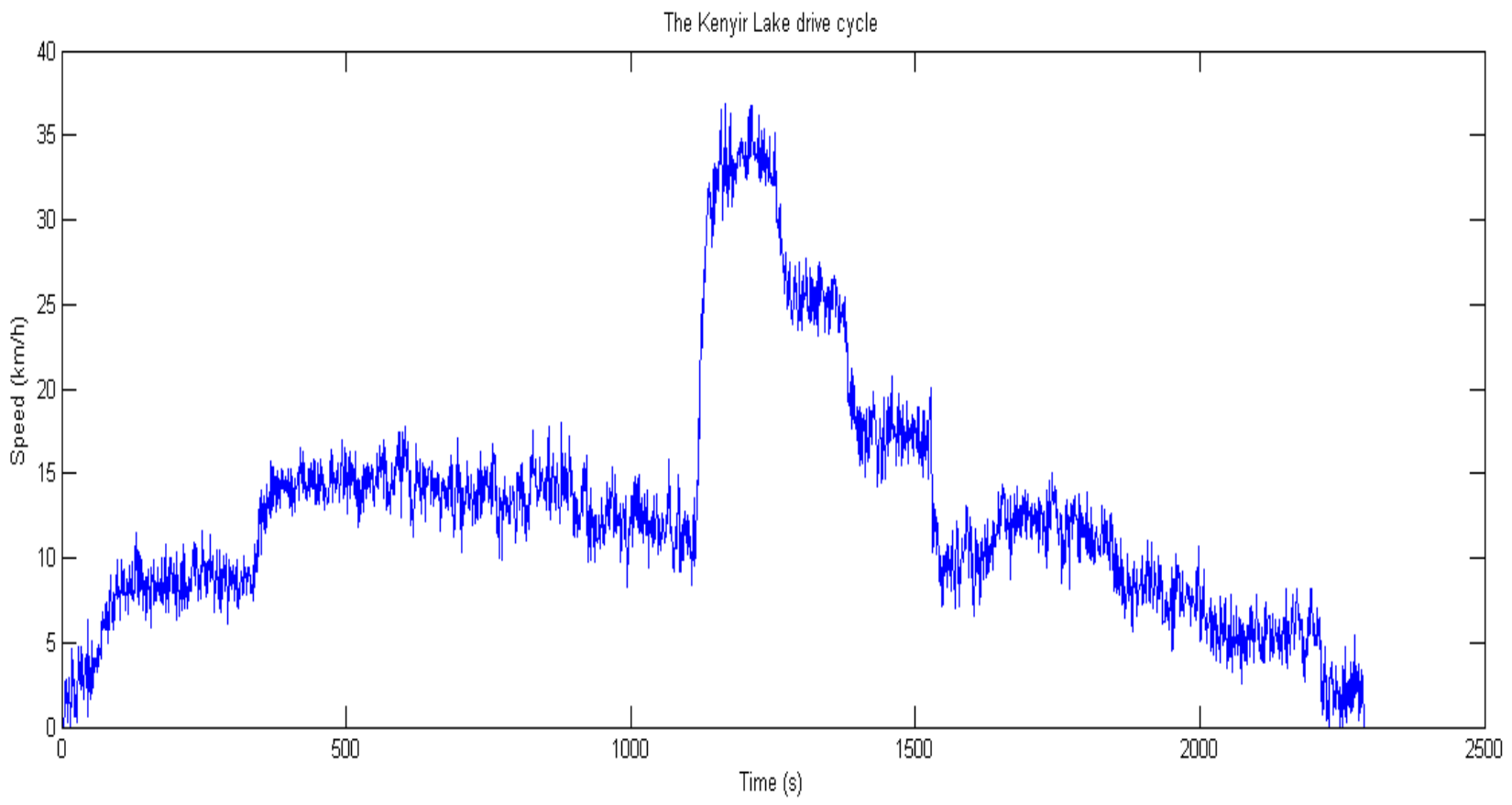

Figure. 4. The Kenyir Lake drive cycle

\section{Conclusion}

Based on the results of this study, it can be concluded that the proposed method is possible to generate a KL driving cycle for PHERB powertrain in order to measure fuel consumption and emissions and it was proved by the tabulate data above. This paper also proved that from executed research above that there is potential ability to use this data on PHERB powertrain.

\section{Acknowledgment}

The financial support of this work by the Fundamental Research Grant Scheme and the Universiti Malaysia Terengganu, is gratefully acknowledged.

\section{References}

1. Kamble, S.H., Mathew, T.V., \& Sharma, G. (2009).
Development of real world driving cycle: Case study of Pune, India. Transportation Research Part D, 132-140.

2. W.T. Hung, H.Y.Tong, C.P. Lee, K.Ha, \& L.Y. Pao. (2007). Development of a Practical Driving Cycle Construction Methodology: A Case Study in Hong Kong. Transportation Research Part D, Vol. 12, pp. 115-128.

3. Z. Xiao, Z. D. Jia and S. J. Min. (2012) A Synthesis of Methodologies and Practices for Developing Driving Cycles. Energy Procedia 16, pp. 1868-1873.

4. Hung, W.T., Tong, H.Y., Lee, C.P., Ha, K., \& Pao, L.Y. (2007).Development of a practical driving cycle construction methodology: A case study in Hong Kong. Transportation Research Part D, 132-140.

5. T. J. Barlow, S. Latham, I. S. McCrae and P. G. Boulter, "A Reference Book of Driving Cycles for Use in the Measurement of Road Vehicle Emissions", published project report PPR354 for the Department for Transport, version 3, June 2009. 